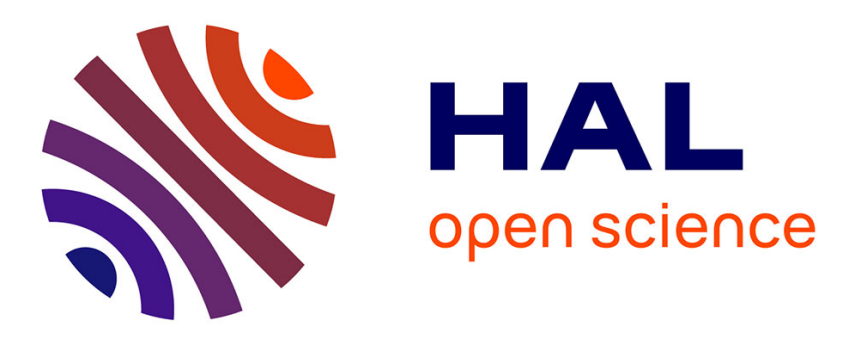

\title{
Any ground associative-commutative theory has a finite canonical system
}

Paliath Narendran, Michaël Rusinowitch

\section{To cite this version:}

Paliath Narendran, Michaël Rusinowitch. Any ground associative-commutative theory has a finite canonical system. [Research Report] RR-1324, INRIA. 1990, pp.11. inria-00075236

\section{HAL Id: inria-00075236 \\ https://hal.inria.fr/inria-00075236}

Submitted on 24 May 2006

HAL is a multi-disciplinary open access archive for the deposit and dissemination of scientific research documents, whether they are published or not. The documents may come from teaching and research institutions in France or abroad, or from public or private research centers.
L'archive ouverte pluridisciplinaire HAL, est destinée au dépôt et à la diffusion de documents scientifiques de niveau recherche, publiés ou non, émanant des établissements d'enseignement et de recherche français ou étrangers, des laboratoires publics ou privés. 


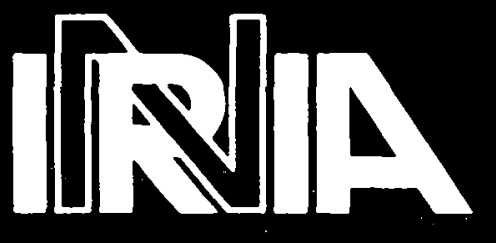

UNITÉ DE RECHERCHE INRIA-LORRAINE

\section{Rapports de Recherche}

\section{$N^{\circ} 1324$}

\section{Programme 1}

Programmation, Calcul Symbolique

et Intelligence Artificielle

\section{ANY GROUND \\ ASSOCIATIVE-COMMUTATIVE \\ THEORY HAS A FINITE \\ CANONICAL SYSTEM}

Institut National de Recherche en Informatique et en Automatique

Domaine de Voluceau Rocquencourt B.P.105

78153 Le Chesnay Cedex

France

Tél.:(1)39635511 

Any ground associative-commutative theory has a finite canonical system

Toute théorie associative-commutative close admet un système de réécriture canonique fini

\author{
Paliath Narendran \\ Michaël Rusinowitch \\ University at Albany, \\ INRIA \& CRIN, \\ State University of New York 615 rue du Jardin Botanique, BP 101, \\ Albany, New York 12222, USA, 54600 Villers-les-Nancy, FRANCE \\ dran@cs.albany.edu \\ rusi@loria.fr
}

\begin{abstract}
We show that theories presented by a set of ground equations and with several associativecommutative symbols always admit a finite canonical system. In particular, the result is obtained through the construction of a reduction ordering which is AC-compatible and total on the set of congruence classes generated by the associativity and commutativity axioms. Such orderings are fundamental for deriving complete theorem proving strategies with builtin associative commutative unification.
\end{abstract}

Résumé

Nous montrons que les théories présentées par un ensemble d'équations closes admettent toujours un système de réécriture canonique fini. Le résultat est obtenu par la construction d'un ordre de réduction $\mathrm{AC}$-compatible et total sur l'ensemble des classes de congruences modulo les axiomes d'associativité et de commutativité. Cet ordre est une extension de la méthode classique des interprétations polynômiales. 


\title{
Any ground associative-commutative theory has a finite canonical system
}

\author{
Paliath Narendran \\ University at Albany, LI-67A \\ State University of New York \\ Albany, New York 12222 \\ USA \\ dran@cs.albany.edu
}

\author{
Michaël Rusinowitch \\ INRIA \& CRIN \\ BP 239 \\ 54506 Vandoeuvre-les-Nancy \\ France \\ rusi@loria.fr
}

\begin{abstract}
We show that theories presented by a set of ground equations and with several associative-commutative symbols always admit a finite canonical system. The methods for obtaining this result are original and we feel that they should have further applications. In particular, the result is obtained through the construction of a reduction ordering which is $\mathrm{AC}$-compatible and total on the set of congruence classes generated by the associativity and commutativity axioms. Such orderings are fundamental for deriving complete theorem proving strategies with built-in associative commutative unification as well as unfailing completion procedures.
\end{abstract}

\section{Introduction}

In this paper, we show that there is an algorithm which, given any finite set $E$ of ground equations which contains associative commutative operation symbols, produces a reduced canonical rewriting system $R$ equivalent to $E$. The method we use follows the general approach of Knuth and Bendix [KB70] of generating word problem decision algorithms for abstract algebras. It has been known for a long time that this method always succeeds with purely ground equations, due to the existence of reduction orderings which are total on ground terms. In order to deal with non orientable axioms, like the commutativity one for instance, the completion technique of Knuth and Bendix has been extended to equivalence class term rewriting systems by [Hue80,LB77,PS81,JK86]. In this framework, Ballantyne and Lankford [BL81] have designed a completion algorithm for finitely presented commutative semigroups. This is the case of one binary commutative-associative function and a finite number of constants. Termination of the algorithm was obtained by Dickson's lemma. Our result can be viewed as a generalization of Ballantyne and Lankford's when there are several binary commutative-associative functions and also non constant function symbols. To prove that completion always succeeds we first need to build a 
reduction ordering which is total on the set of classes of terms with respect to the congruence generated by the associative commutative axioms. This construction, which generalizes polynomial orderings, is performed in Section 3. Then, in Section 4, we define a completion procedure and show that it always stops with a finite canonical system. Here, as in the semigroup case, Dickson's lemma is the decisive argument.

\section{Preliminaries and notations}

Here, we suppose that we are given a signature $F$ which contains a subset $F_{A C}$ of associative commutative operations. Our notations will be adapted to this framework. For a detailed survey on term rewriting the reader may consult [DJ90].

Let $T(F)$ be the set of terms on $F$ (all terms to be considered in the sequel do not have variables). The congruence on $T(F)$ generated by the associative commutative equations satisfied by the symbols in $F_{A C}$ will be written: $=F_{A C}$ or $=_{A C}$ when there is no ambiguity. The extension of this relation to multisets of terms is $=={ }_{A C}$. The root symbol of a term $t$ is denoted by $\operatorname{root}(t)$. We write $s[t]_{p}$ to indicate that a term $s$ contains $t$ as a subterm at position $p$. Positions are classically coded by sequences of integers. For instance, $t_{\mid p}$ indicates the subterm of $t$ which occurs at position $p$.

A rewrite rule on $T(F)$ is an ordered pair $(l, r)$ of terms also denoted by $l \rightarrow r$. A rewrite system is any set $R$ of rewrite rules. The rewriting relation generated by a rewrite system $R$ is the binary relation $\rightarrow_{R}$ defined on terms by: $s \rightarrow_{R} t$ iff $s={ }_{A C} u\left[\eta_{p}\right.$ and $t={ }_{A C} u[r]_{p}$ for some context $u$, position $p$ and rule $l \rightarrow r$ in $R$. The inverse of $\rightarrow_{R}$ is denoted by $\leftarrow_{R}$. The transitive reflexive closure of $\rightarrow_{R}$ (resp. $\leftarrow_{R}$ ) is denoted by $\rightarrow_{R^{*}}$ (resp. $\leftarrow_{R^{*}}$ ). A term $t$ is irreducible in $R$ if there is no $u$ such that $t \rightarrow_{R^{u}}$. An irreducible term $s$ is called a normal

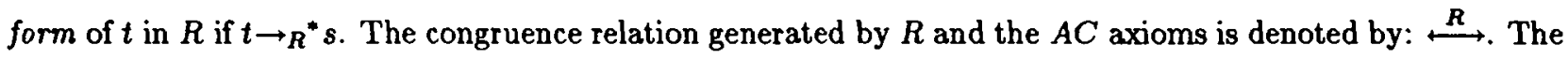
rewrite system $R$ is confluent if $s \leftarrow R^{*} u \rightarrow_{R^{*} t}$ implies that there exists $v$ such that $s \rightarrow R^{*} v \leftarrow_{R}^{*} t$. The rewrite system $R$ is locally confluent if $s \leftarrow_{R} u \rightarrow_{R} t$ implies that there exists $v$ such that $s \rightarrow_{R}^{*} v \sim_{R}^{*} t$. A rewrite system $R$ terminates if $\rightarrow_{R}$ is noetherian. A set $R$ of rules is reduced if for every rule $l \rightarrow r$ in $R$, both $l$ and $r$ are irreducible under $\rightarrow_{R-\{l \rightarrow r\}}$. A rewrite system is canonical if it is terminating, confluent and reduced. In a canonical system terms have unique normal forms. As a consequence the word problem is decidable in every theory which admits a canonical system.

\section{A compatible ordering for associative-commutative theories}

The design of orderings for proving termination of rewrite systems modulo $A C$ has been considered as a hard task. In fact, to our knowledge, very few constructions are available in the literature. Perhaps the best known among them is the associative path ordering scheme [BP85], which extends the recursive path ordering (see also [BD86]). However, this ordering puts serious limitations on the precedence of AC-symbols. In fact two ACsymbols cannot be compared in the precedence unless they are related by a distributivity law. That explains why there is no hope to get a total ordering from the associative path ordering. 
Another construction uses polynomial interpretations [Lan79,BL87]. We are going to elaborate on this method in order to obtain an ordering with the required properties for our purpose. Hence, let us recall the interpretation technique. Each $n$-ary function symbol $f$ is interpreted as an integer polynomial $P^{f}$ with $n$ indeterminates. The interpretation $I(t)$ of a term $t$ is recursively defined by the rule:

$$
I\left(f\left(t_{1}, \ldots, t_{n}\right)\right)=P^{f}\left(I\left(t_{1}\right), \ldots, I\left(t_{n}\right)\right)
$$

A set of rules terminates if we can choose polynomial interpretations such that for every rule $l \rightarrow r$ in the set we have $I(l)>I(r)$. Some other properties, to be detailed in this section, are also required.

For proving termination of rewrite systems modulo $\mathrm{AC}$ axioms, the ordering should possess the $A C$ compatibility property:

Definition 1 An ordering $>$ on $T(F)$ is $A C$-compatible iff whenever we have $s>t, s={ }_{A C} s^{\prime}$ and $t={ }_{A C} t^{\prime}$ we also have $s^{\prime}>t^{\prime}$.

The easiest way to ensure AC-compatibility (when dealing) with polynomial interpretations is to use polynomials which interpret identically the terms of the same AC-class. BenCherifa and Lescanne have pointed out the following necessary and sufficient condition for such a property:

Each polynomial $P^{f}(x, y)$ interpreting an $A C$ symbol $f$ must be of the form axy $+b(x+y)+c$ where the coefficients satisfy $b^{2}=b+a c$.

We have been unable to use these classical polynomial interpretations in order to get an ordering which is also monotonic and total. Therefore we shall use another construction which may be interesting from its own. Instead of taking numerical values for the coefficients $a, b$ and $c$, we shall take integer polynomials for them. Let us describe precisely the construction.

Our interpretation domain will be the free commutative ring on $\left\{X_{f} ; f \in F\right\}$, which is isomorphic to $Z\left[X_{1}, \ldots, X_{m}\right]$, where $m$ is the cardinal of $F$. This algebra will be denoted by $Z T$. Hence, to each function symbol $f \in F$, is associated an indeterminate $X_{f}$.

The subset of elements of $Z T$ whose coefficients are non-negative integers is denoted by $N T$. Let us define an ordering on $N T$. We suppose given a total precedence on the indeterminates and we suppose that the indeterminates of every monomial are sorted decreasingly according to this order. Two monomials are compared first by their degree, and second, when these degrees are equal, by lexicographic order.

We define $>_{N}$ to be the multiset extension of this ordering and we shall use it to compare polynomials, by treating them as multiset of monomials. The main property of $>_{N}$ is that it is well-founded.

The set $N T$ is the target of the interpretation I that we are going to introduce now. For every term $f\left(t_{1}, \ldots, t_{n}\right)$ in $T(F)$,

$$
I\left(f\left(t_{1}, \ldots, t_{n}\right)\right)=\left(X_{f}+1\right)\left(\left(X_{f}^{2}+2 X_{f}\right) I\left(t_{1}\right) I\left(t_{2}\right) \cdots I\left(t_{n}\right)+\left(X_{f}+1\right)\left(I\left(t_{1}\right)+I\left(t_{2}\right)+\cdots+I\left(t_{n}\right)\right)+1\right)
$$

and for any constant $a, I(a)=X_{a}+1$. 
The following lemma is obtained by an easy computation:

Lemma 1 If $s={ }_{A C} t$ then $I(s)=I(t)$.

Let $\operatorname{root}(t)$ denote the root symbol of a term $t$. When the arity of $\operatorname{root}(t)$ is $k$, the $k$-uple $\left(t_{11}, \ldots, t_{\mid k}\right)$ of immediate subterms of $t$ is denoted by $i m(t)$. Let us also introduce for each AC-symbol $f$ a function $\rho_{f}$ which applies every term to a multiset of terms and which is defined recursively as:

$$
\rho_{f}\left(g\left(s_{1}, \ldots s_{n}\right)\right)=\left\{\begin{array}{lc}
\bigcup_{i=1}^{i=n} \rho_{f}\left(s_{i}\right) & \text { if } g=f \\
\left\{g\left(s_{1}, \ldots s_{n}\right)\right\} & \text { otherwise }
\end{array}\right.
$$

The next lemma tells that we can recover the root symbol of $s$ from its interpretation $I(s)$.

Lemma 2 Given terms $s$ and $t$, if $I(s)=I(t)$ then $\operatorname{root}(s)=\operatorname{root}(t)$.

Proof: Suppose that $s=f\left(s_{1}, \ldots, s_{n}\right)$ and $t=g\left(t_{1}, \ldots, t_{m}\right)$, where $f$ and $g$ represent two different function symbols. Then

$$
\begin{aligned}
& I(s)=\left(X_{f}+1\right)\left(\left(X_{f}^{2}+2 X_{f}\right)\left(I\left(s_{1}\right) \cdots I\left(s_{n}\right)\right)+\left(X_{f}+1\right)\left(I\left(s_{1}\right)+\cdots+I\left(s_{n}\right)\right)+1\right) \\
& I(t)=\left(X_{g}+1\right)\left(\left(X_{g}^{2}+2 X_{g}\right)\left(I\left(t_{1}\right) \cdots I\left(t_{m}\right)\right)+\left(X_{g}+1\right)\left(I\left(t_{1}\right)+\cdots+I\left(t_{m}\right)\right)+1\right)
\end{aligned}
$$

If $I(s)=I(t)$ then $\left(X_{f}+1\right)$ divides $I(t)$ in $Z T$. However, since $Z T$ is a factorial ring and $\left(X_{f}+1\right)$ does not divide $\left(X_{g}+1\right)$, we have:

$$
\left(X_{f}+1\right) \mid\left(\left(X_{g}^{2}+2 X_{g}\right)\left(I\left(t_{1}\right) \cdots I\left(t_{m}\right)\right)+\left(X_{g}+1\right)\left(I\left(t_{1}\right)+\cdots+I\left(t_{m}\right)\right)+1\right)
$$

Let $g_{1}, \ldots, g_{m}$ be the root symbols of $t_{1}, \ldots, t_{m}$ respectively. Applying the substitution $\sigma=\left\{X_{g} \leftarrow X_{f}, X_{g_{2}} \leftarrow\right.$ $\left.X_{f}, \ldots, X_{g_{m}} \leftarrow X_{J}\right\}$ to the previous expression, we get:

$$
\left(X_{f}+1\right) \mid\left(\left(X_{f}^{2}+2 X_{f}\right) \sigma\left(I\left(t_{1}\right) \cdots I\left(t_{m}\right)\right)+\left(X_{f}+1\right) \sigma\left(I\left(t_{1}\right)+\cdots+I\left(t_{m}\right)\right)+1\right)
$$

This is equivalent to have:

$$
\left(X_{f}+1\right) \mid X_{f} \cdot \sigma\left(I\left(t_{1}\right) \cdots I\left(t_{m}\right)\right)+1
$$

However, since every $I\left(t_{i}\right)$ admits some $X_{g_{k}}+1$ as a factor, we can notice that $X_{f}+1$ divides $\sigma\left(I\left(t_{1}\right) \cdots I\left(t_{m}\right)\right)$. This rises a contradiction. $\square$

Lemma 3 Given terms $s$ and $t$, if $I(s)=I(t)$ then every function symbol has the same number of occurrences in $s$ and $t$.

Proof: Notice that the maximal monomial in $I(s)$ is $\prod_{f \in S} X_{f}^{3} \prod_{f \in C} X_{f}$ where $S$ (resp. $C$ ) is the multiset of symbols in $s$ whose arity is $>0$ (resp. 0 ).

We are now in position to define the relation on $T(F)$ that we want to use as an ordering for proving termination.

Definition 2 Let $s=f\left(s_{1}, \ldots, s_{n}\right)$ and $t=g\left(t_{1}, \ldots, t_{m}\right)$. Then $s \succ t$ iff 
- $I(s)>_{N} I(t)$ or

- $I(s)=I(t)$ and

- if $f \in A C$ then $\rho_{f}(s) \succcurlyeq \rho_{f}(t)$

- if $f \notin A C$ then $\operatorname{im}(s) \succ_{\operatorname{lex}} \operatorname{im}(t)$

where $\succcurlyeq$ is defined by: $X=\left\{a_{1}, \ldots, a_{n}\right\} \succcurlyeq Y=\left\{b_{1}, \ldots, b_{m}\right\}$ iff

$-X \neq \emptyset$ and $Y=\emptyset$ or

- for some $i, j: a_{i}={ }_{A C} b_{j}$ and $X-\left\{a_{i}\right\} \nsucc Y-\left\{b_{j}\right\}$ or

- for some $i, j_{1}, \ldots, j_{k}: a_{i} \succ b_{j_{1}}, \ldots, b_{j_{k}} X-\left\{a_{i}\right\} \succ Y-\left\{b_{j_{1}}, \ldots, b_{j_{k}}\right\}$.

- for some $i, j_{1}, \ldots, j_{k}: a_{i} \succ b_{j_{1}}, \ldots, b_{j_{k}} X-\left\{a_{i}\right\}==_{A C} Y-\left\{b_{j_{1}}, \ldots, b_{j_{k}}\right\}$.

and where $\succ_{\text {lex }}$ is defined by: $\left(a_{1}, \ldots, a_{n}\right) \succ_{l e x}\left(b_{1}, \ldots, b_{m}\right)$ iff $\exists i, \forall j<i a_{j}=_{A C} b_{j}$ and $a_{i} \succ b_{i}$.

The important properties of this relation are quoted in the next proposition:

\section{Proposition 1 The relation $\succ$ is}

1. irreflexive and transitive

2. well-founded (there is no infinite anti-chain $t_{1} \succ t_{2} \succ \ldots$ )

3. monotonic (for any function $f$ and terms $s, t, s_{1}, \ldots, s_{n}, s \succ t$ implies $s^{\prime}=f\left(s_{1}, \ldots, s_{i-1}, s, s_{i+1}, \ldots, s_{n}\right) \succ$ $\left.f\left(s_{1}, \ldots, s_{i-1}, t, s_{i+1}, \ldots, s_{n}\right)=t^{\prime}\right)$.

4. total on the set of $A C$-classes (for all $s, t$ we have either $s \succ t$ or $t \succ s$ or $s={ }_{A C} t$ )

5. AC-compatible.

Proof of 1: Irreflexivity is obtained easily by induction on the size of terms. Suppose now that $t \succ s$ and $s \succ u$. If $I(t)>_{N} I(s)$ and $I(s)>_{N} I(u)$ then $I(t)>_{N} I(u)$ and therefore $t \succ u$. We can draw the same conclusion if $I(t)=I(s)$ and $I(s)>_{N} I(u)$ or if $I(t)>_{N} I(s)$ and $I(s)=I(u)$. Now, if $I(t)=I(s)=I(u)$ then, due to lemma $2, s, t$, and $u$ have the same root symbol $f$. Suppose $f$ is AC then $\rho_{f}(t) \nrightarrow \rho_{f}(s)$ and $\rho_{f}(s) \succcurlyeq \rho_{f}(u)$. By induction on the sizes of terms we have $\rho_{f}(t) \succcurlyeq \rho_{f}(u)$ and then $t \succ u$. When $f$ is not AC, we apply the same reasoning to $\succ_{l e x}$.

Proof of 2: Suppose that there is an infinite antichain: $t_{1} \succ t_{2} \succ \ldots$. Since $>_{N}$ is well-founded, there exists $k$ such that for all $j \geq k$, we have $I\left(t_{j}\right)=I\left(t_{k}\right)$. Hence, by lemma 3 , the terms $t_{j}$ for $j \geq k$ have the same number of occurrences of any function symbol. But from a finite multiset of symbols we can build only finitely many ground terms. Therefore, by the pigeon-hole principle, there exist two indices $l, m(l>m)$ such that $t_{l}=t_{m}$. But, by transitivity, $t_{l} \succ t_{m}$. However, this is not compatible with irreflexivity.

Proof of 3: If $I(s)>_{N} I(t)$ then $I(f(. . s))>._{N} I(f(. t .)$.$) and f(. . s ..) \succ f(. t .$.$) follows. Suppose now$ that $I(s)=I(t)$. If $f$ is not an AC symbol, $i m\left(s^{\prime}\right) \succ_{\text {lex }} i m\left(t^{\prime}\right)$. If $f$ is an AC symbol then we notice that $\rho_{f}\left(s^{\prime}\right) \nrightarrow \rho_{f}\left(t^{\prime}\right)$. 
Proof of 4: We use induction on the size of terms. Suppose that 4 is true for all terms of size $<k$. Then n-uples (resp. multisets) of terms each of size less than $k$ can be ordered with $\succ_{\text {lex }}$ (resp. $\nsucc$ ). Let $s, t$ be such that (at least) one of them has size $k$. Suppose that neither $s \succ t$ nor $t \succ s$. Then $I(s)=I(t)$. But, if $f=\operatorname{root}(s)$ is $\mathrm{AC}$, by induction hypothesis, $\rho_{f}(s) \gg \rho_{f}(t)$ or $\rho_{f}(t) \succ \rho_{f}(s)$ or $i m(s)==A C i m(t)$. The first two cases contradict the hypothesis. Hence there only remains the third from which we derive $s={ }_{A C} t$. When $\operatorname{root}(s)$ is not $\mathrm{AC}$, the proof is similar.

Proof of 5: Suppose that $s^{\prime}={ }_{A C} s=f\left(s_{1}, \ldots, s_{n}\right) \succ t=g\left(t_{1}, \ldots, t_{n}\right)={ }_{A C} t^{\prime}$. If $I(s)>_{N} I(t)$, by lemma 1 , we also have $I\left(s^{\prime}\right)>_{N} I\left(t^{\prime}\right)$. If $I(s)=I(t)$ and $f$ is not an $\mathrm{AC}$ function, the corresponding components of im(s) and $i m\left(s^{\prime}\right)$ are AC-equal. Since $g=f$, the same is true for $i m(t)$ and $i m\left(t^{\prime}\right)$. Therefore, $i m\left(s^{\prime}\right) \succ_{\operatorname{lex}} i m\left(t^{\prime}\right)$. If $f$ is $\mathrm{AC}$, notice that $\rho_{f}(s)==_{A C} \rho_{f}\left(s^{\prime}\right)$ and $\rho_{f}(t)===_{A C} \rho_{f}\left(t^{\prime}\right)$. From $\rho_{f}(s)==_{A C} \rho_{f}(t)$, we derive also $\rho_{f}\left(s^{\prime}\right)=={ }_{A C} \rho_{f}\left(t^{\prime}\right)$.

\section{Completion of ground systems with associative-commutative symbols}

Solving the word problem by completion in theories presented by sets of ground equations is known to be always possible, even efficiently [GNP*88,Sny89]. When the signature is built solely from constants and one associative commutative operation, we get finitely presented commutative semigroups. In such structures, the word problem can still be solved by completion [BL81]. We are going to show in the following that this method generalizes to signatures which contain free function symbols and several associative commutative operations.

For convenience, we shall use the flattened representation for terms which contain AC symbols. For instance, if $f \in F_{A C}$, a flattened representation of $f(a, f(b, c))$ is $f(a, b, c)$. When $f\left(t_{1}, \ldots, t_{n}\right)$ is a flattened representation of $t$ and $f \in A C$, then for all $i$ the root of $t_{i}$ differs from $f$. In that case, we say that the $t_{i}$ are the fundamental subterms of $t$ and we write $f s(t)=\left\{t_{1}, \ldots, t_{n}\right\}$. Note also that $\rho_{f}(t)=f s(t)$ when the root of $t$ is $f \in F_{A C}$.

In the completion approach to word problems, the main operation is generation of critical pairs, which can be used to test the confluence property of the rewriting relation. Hence we give the suitable definition of critical pairs for our framework:

Definition 3 Assume that $l \rightarrow r$ and $g \rightarrow d$ are two rules in $R$ such that $l={ }_{A C} f\left(t_{1}, \ldots, t_{n}\right), g=A C$ $f\left(s_{1}, \ldots, s_{m}\right), f \in F_{A C}$ and there exist $i$ and $j$ such that $t_{i}={ }_{A C} s_{j}$. Let $S$ be a maximal multiset of terms such there exist $\left\{k_{1}, \ldots, k_{v}\right\} \subseteq\{1, \ldots, n\}$ and $\left\{l_{1}, \ldots, l_{u}\right\} \subseteq\{1, \ldots, n\}$ with:

$$
S \cup\left\{t_{k} ; k \in\left\{k_{1}, \ldots, k_{v}\right\}\right\}=={ }_{A C}\left\{t_{k} ; k=1, . ., n\right\}
$$

and

$$
S \cup\left\{s_{l} ; l \in\left\{l_{1}, \ldots, l_{u}\right\}\right\}=={ }_{A C}\left\{s_{l} ; l=1, \ldots, m\right\}
$$

The pair of terms $\left(f\left(s_{l_{1}}, \ldots, s_{l_{v}}, r\right), f\left(t_{k_{1}}, \ldots, t_{k_{v}}, d\right)\right)$ is called a critical pair. 
A critical pair $(r, s)$ of $R$ is trivial if there is a term $t$ such that $r \rightarrow R^{*} t$ and $s \rightarrow R^{*} t$. The main property of reduced systems of rules is stated in the proposition:

Proposition 2 Given a reduced system $R$, the relation $\rightarrow_{R}$ is locally confluent if and only if every critical pair $(r, s)$ of $R$ is trivial.

Remark: If we compare with the general AC-completion procedure of [PS81], we find that the only critical pairs we need here are between a rule and an extended rule. There are no critical pairs between rules since the left-hand side are kept reduced. Critical pairs between extended rules are covered by the other ones.

We introduce now a completion procedure which given a ground AC theory always yields a canonical system. We assume that the equations are oriented according to an ordering $>$ which is well-founded, monotonic, $A C$ compatible and total on the set of $\mathrm{AC}$ congruence classes. Due to our construction of Section 3, we know that such orderings exist. Our completion procedure when applied to an initial set $L_{0}$ of rules produces a sequence of sets of rules $L_{0}, R_{0}, \ldots, L_{i}, R_{i}, L_{i+1}, R_{i+1}, \ldots$. which is defined as follows:

- Given $L_{i}$, generate a reduced set $R_{i}$ :

- While possible do:

* Choose $(r, s)$ and simplify $R_{i}-\{(r, s)\}$ with it.

* For any simplified rule $(u, v)$ do

If $u={ }_{A C} v$ then delete $(u, v)$ else if $v>u$ then replace $(u, v)$ by $(v, u)$.

- EndWhile

- Given $R_{i}$, compute its set of non-trivial critical pairs $C P_{i}$.

- If $C P_{i}=\emptyset$ then halt else let $L_{i+1}=R_{i} \cup\left\{(r, s) ;(r, s)\right.$ or $(s, r) \in C P_{i}$ and $\left.r>s\right\}$.

Given a set of rules $R$, and an $A C$ symbol $f$, we define its set of generators $G_{f}(R)$ to be the set of congruence classes modulo $R$ of the elements of the following set:

$$
\bigcup_{(l, r) \in R_{f}}\left(\rho_{f}(l) \cup \rho_{f}(r)\right)
$$

where $R_{f}$ is the set of rules in $R$ such that at least one of their sides has $f$ as root symbol. We also define $N(R)$ to be the set of sides of rules of $R$ whose root symbol is not AC. For instance, consider the following system $R$ :

$$
\begin{aligned}
a+b & \rightarrow a * b \\
a * c & \rightarrow g(e) \\
e & \rightarrow f
\end{aligned}
$$

where + and $*$ are AC. Then $G_{+}(R)=\{a, b, a * b\}, G_{*}(R)=\{a, b, c, a+b, g(e)\}$ and $N(R)=\{e, f, g(e)\}$. The elements of $G_{+}(R)$ and $G_{*}(R)$ have to be considered as representative of their congruence classes modulo $R$. 
The important fact about the generators set is that it cannot increase during the completion procedure.

Let us be more specific. The relation $>$ is extended to sets of terms in the following way: $A>B$ if $B$ is obtained either by replacing some element $a$ of $A$ by $b$ such that $a>b$ or by removing some element from $A$. The relation $>$ is still well-founded on sets. Due to the structure of our completion procedure which simplifies all occurrences of a reducible term in the same step, we notice that $N(R)$ cannot get larger with respect to $>$.

Lemma 4 For all $i \geq 0, G_{f}\left(R_{i}\right) \subseteq G_{f}\left(L_{i}\right)$ and $N\left(R_{i}\right) \leq N\left(L_{i}\right)$. If $(r, s)$ is a critical pair of $R$ with $r>s$, then $G_{f}(R \cup\{(r, s)\})=G_{f}(R)$ and $N(R)=N(R \cup\{(r, s)\})$.

Theorem 1 When applied to any finite set of rules $R$, the completion procedure halts with a canonical system.

Proof: The procedure never fails to orient a new pair of terms since we are provided with an ordering which can always compare two terms which are not congruent modulo AC.

We extend $>$ to rewrite rules by comparing their left-hand sides and right-hand sides in a lexicographic way. This ordering on rules is noetherian. We shall assume that the following slight restriction is imposed to the control of the completion procedure :

Hypothesis: a rule is never reduced by a bigger rule.

Consider now the smallest rule $r_{1}$ in $\bigcup_{i \geq 0} R_{i}$. It belongs to some set $R_{i_{1}}$. Let us notice that $r_{1}$ also belongs to any $R_{j}$ with $j \geq i_{1}$. Otherwise it would be reducible by some other rule in $\bigcup_{i \geq 0} R_{i}$. But this possibility is ruled out by the fact that $r_{1}$ is minimal. Consider now the rule $r_{2}$ which is minimal in $\left(\bigcup_{i \geq i_{1}} R_{i}\right)-\left\{r_{i_{1}}\right\}$. It belongs to some set $R_{i_{2}}$. Note that $r_{2}$ also belongs to any $R_{j}$ with $j \geq i_{2}$. The reason is that if it were reducible, that could only be possible by $r_{1}$. However, since $R_{i_{2}}$ is reduced this case is excluded.

Hence, by induction, we can build a sequence $r_{i_{1}}, \ldots, r_{i_{k}}, \ldots$ such that $r_{i_{k}}$ is minimal in $\left(\bigcup_{i \geq i_{k-1}} R_{i}\right)-$ $\left\{r_{i_{1}}, \ldots, r_{i_{k-1}}\right\}$. The fact that $R_{i_{k}}$ is reduced shows that $r_{i_{k}}$ cannot be reduced later on. Consider now the set $S=\left\{r_{i_{1}}, \ldots, r_{i_{k}}, \ldots\right\}$. We have the following result:

Lemma 5 The set of rules $S$ is canonical.

Sketch of proof. It is not difficult to see that $S$ is reduced. Suppose that there is a non-trivial critical pair $(u, v)$ between two rules of $S$, say $r_{l}$ and $r_{m}$. Consider a minimal proof of $(u, v)$ in $\bigcup_{i \geq 0} R_{i}$ with respect to the proof ordering defined in [BDH86]. If one step uses a rule which is not in $S$, this rule will be reduced at some stage of the algorithm. Therefore we can build a smaller proof. Hence, we can assume that every step in the minimal proof of $(u, v)$ is justified by a rule in $S$. If this proof is not a rewrite proof, then the computation of a new critical pair allows again to derive a smaller proof.

Let us prove now that $S$ is finite. Assume that $S$ is infinite. We notice that for any AC symbol $f$, $G_{f}(S) \subseteq G_{f}(R)$. Hence, $G_{f}(S)$ is finite. Also by lemma 4 we can prove that $N(S)$ is finite. Since $S$ is infinite there are infinitely many rules $\left(l_{i} \rightarrow r_{i}\right)_{i \geq 0}$ whose left-hand sides have the same root symbol $f$. From the finiteness of $N(S)$ we deduce that $f$ is necessarily AC. Since $S$ is reduced, all left-hand sides of $S$ are in normal form. As a consequence, we have: 
Lemma 6 If $s \in f s\left(l_{i}\right), t \in f s\left(l_{j}\right)$ and $s \stackrel{R}{\longrightarrow} t$ then $s=A C t$.

Let us introduce the notation $L_{i}$ for the multiset of congruence classes modulo $\stackrel{R}{\longrightarrow}$ of the elements of $f s\left(l_{i}\right)$. Since $G_{f}(S)$ is finite (its elements are supposed numbered from 1 to $\mathrm{M}$ ) and since every element of $L_{i}$ is also an element of $G_{f}(S)$ then we can associate to each $L_{i}$ a vector of integers $\left(h_{1}, h_{2}, \ldots, h_{M}\right)$ where $h_{g}$ is the number of times that the element of rank $g$ in $G_{f}(S)$ occurs in $L_{i}$. We are going to apply Dickson's lemma [Dic13] to this set of vectors. Let us recall this result:

Lemma 7 Given $n \in N$, every infinite sequence of $n$-dimensional vectors with nonnegative integer components must contain an infinite subsequence that is nondecreasing with respect to $\leq$ where $W \leq V$ if there exists a vector $U$ with nonnegative components such that $V=W+U$.

From this lemma we deduce that there exist two rules $l_{k} \rightarrow r_{k}$ and $l_{j} \rightarrow r_{j}$ such that $L_{k}$ is a submultiset of $L_{j}$. By the previous lemma, this ensures the existence of a submultiset $A$ of $f s\left(l_{j}\right)$ such that $A==A C f s\left(l_{k}\right)$. This implies that $l_{j}$ can be simplified by $l_{k} \rightarrow r_{k}$, in contradiction with the fact that $S$ is reduced.

We can now achieve the proof of theorem 1 . Since $S$ is finite, let $m$ be the smallest index such that $S \subseteq R_{m}$. One easily sees that $S=R_{j}$ for all $j \geq m$. In particular $R_{m+1}=R_{m}$, which means that the completion procedure stops at step $m$.

\section{Related problems, further works and conclusion}

In the previous section, it was shown that in ground associative commutative theories the word problem can always be solved by construction of a canonical system. A natural generalization of the word problem is the unifiability problem. We have investigated this problem by coding it as a reachability problem in Petri nets [PM90]. Then from the decidability of reachability in Petri nets [May81], the decidability of unifiability in ground AC theories should follow. This idea comes from the observation that the elements of finitely presented commutative semigroups can be represented as vectors of integers. In the general case of several associative commutative symbols, the problem must be coded as a conjunction of reachability problems, each of them being related to one of the AC symbols.

For proving that we can always orient ground AC systems we have developed a generalization of the polynomial ordering. Instead of using polynomials with numerical range we rather use polynomials whose range are polynomial rings. To our knowledge, this is the first occurrence of a reduction ordering which is total on the set of congruence classes modulo AC and which is AC-compatible (for a signature which contains any number of AC symbols). Ordering with such properties are fundamental to derive refutationally complete theorem-proving strategies with built-in associative commutative unification [AH90]. They should be useful, too, for the design of new unfailing completion procedures. The question remains open whether there exist classical numerical polynomials with the same properties than the ordering we have proposed here.

Another interesting issue would be to see what happens when we replace the associativity and commutativity axioms by another well-behaved theory $E$. Does every ground presentation admit a canonical rewrite system 
modulo $E$ ? An interesting result along these lines is the construction in [KN85] of a Thue system which has a decidable word problem but which has no canonical system.

Acknowledgements: We thank Eric Domenjoud for reading the manuscript.

\section{References}

[AH90] Siva Anantharaman and Jieh Hsiang. An automated proof of the moufong identities in alternative rings. J. Automated Reasoning, 6:79-109, 1990.

[BD86] L. Bachmair and N. Dershowitz. Commutation, transformation and termination. In J. Siekmann, editor, Proceedings 8th Conf. on Automated Deduction, pages 5-20, Springer-Verlag, 1986. Lecture Notes in Computer Science, volume 230.

[BDH86] L. Bachmair, N. Dershowitz, and J. Hsiang. Orderings for equational proofs. In Proceedings Symp. Logic in Computer Science, pages 346-357, Boston (Massachusetts USA), 1986.

[BL81] A.M. Ballantyne and D. Lankford. New decision algorithms for finitely presented commutative semigroups. Comp. \& Maths. with Appl., 7:159-165, 1981.

[BL87] A. BenCherifa and P. Lescanne. Termination of rewriting systems by polynomial interpretations and its implementation. Science of Computer Programming, 9(2):137-160, October 1987.

[BP85] L. Bachmair and D.A. Plaisted. Termination orderings for associative-commutative rewriting systems. Journal of Symbolic Computation, 1:329-349, 1985.

[Dic13] L. Dickson. Finiteness of the odd perfect and primitive abundant numbers with $n$ distinct prime factors. Amer. J. Math., XXXV:413-422, 1913.

[DJ90] N. Dershowitz and J.-P. Jouannaud. Rewrite systems. In Van Leuven, editor, Handbook of Theoretical Computer Science, North Holland, 1990.

[GNP*88] J. Gallier, P. Narandran, D. Plaisted, S. Raatz, and W. Snyder. Finding canonical rewriting systems equivalent to a finite set of ground equations in polynomial time. In E. Lusk and R. Overbeek, editors, Proceedings 9th Int. Conf. on Automated Deduction, pages 182-196, Springer-Verlag, Lecture Notes in Computer Science, 1988.

[Hue80] G. Huet. Confluent reductions : abstract properties and applications to term rewriting systems. Journal of the Association for Computing Machinery, 27(4):797-821, October 1980.

[JK86] J.-P. Jouannaud and H. Kirchner. Completion of a set of rules modulo a set of equations. SIAM Journal of Computing, 15(4):1155-1194, 1986.

[KB70] D.E. Knuth and P.B. Bendix. Simple word problems in universal algebras. In J. Leech, editor, Computational Problems in Abstract Algebra, pages 263-297, Pergamon Press, Oxford, 1970. 
[KN85] D. Kapur and P. Narendran. A finite Thue system with decidable word problem and without equivalent finite canonical system. Theoretical Computer Science, 35:337-344, 1985.

[Lan79] D.S. Lankford. On Proving Term Rewriting Systems are Noetherian. Technical Report, Louisiana Tech. University, Mathematics Dept., Ruston LA, 1979.

[LB77] D. Lankford and A. Ballantyne. Decision procedures for simple equational theories with permutative axioms: complete sets of permutative reductions. Technical Report, Univ. of Texas at Austin, Dept. of Mathematics and Computer Science, 1977.

[May81] E. W. Mayr. An algorithm for the general petri net reachability problem. In Proceedings of STOC, 1981.

[PM90] Narendran P. and Rusinowitch M. Unifiability in ground AC theories. 1990. In preparation.

[PS81] G. Peterson and M. Stickel. Complete sets of reductions for some equational theories. Journal of the Association for Computing Machinery, 28:233-264, 1981.

[Sny89] W. Snyder. Efficient completion: a $O(n \log n)$ algorithm for generating reduced sets of ground rewrite rules equivalent to a set of ground equations E. In N. Dershowitz, editor, Proceedings 3rd Conf. on Rewriting Techniques and Applications, pages -, Springer-Verlag, Lecture Notes in Computer Science, 1989. 

ISSN 0249 - 6399 